\title{
Late-Onset Guillain-Barré Syndrome and Right Facial Nerve Palsy after COVID-19 Infection
}

\author{
Meari Taguchia Kyle Bonner ${ }^{b} \quad$ Anza Bilal Memon ${ }^{a}$ b \\ aDepartment of Neurology, Henry Ford Hospital, Detroit, MI, USA; bWayne State University, \\ School of Medicine, Detroit, MI, USA
}

\section{Keywords}

Acute inflammatory demyelinating polyradiculoneuropathy · COVID-19 · SARS-CoV-2 .

Intravenous immunoglobulin-G · Immune-mediated neuropathy · GBS

\section{Abstract}

Here, we present a case of late-onset Guillain-Barré syndrome (GBS) associated with COVID-19. A 70-year-old woman presented with ascending paralysis and right lower motor neuron facial weakness 2 months after COVID-19 infection. Test results for SARS-CoV-2 immunoglobulin were positive at the time of presentation. Lumbar puncture showed albuminocytological dissociation, and electrophysiology showed features of demyelination with secondary axon loss. In the published literature on GBS associated with COVID-19, almost all patients presented with neurological symptoms 1-4 weeks after the infection. GBS can be an early or late manifestation after COVID-19. Patients with signs of paraparesis and facial weakness after COVID-19 should be carefully evaluated for immune-mediated central and peripheral nervous system disorders.

\section{Introduction}

COVID-19 has left a distinctive mark in the medical and economic landscape of the year 2020. COVID-19 research has boomed recently, including within neurology, since neurological complications from SARS-CoV-2 infection have been reported. Both central and peripheral nervous system involvement have been reported with COVID-19 [1], and several 
cases of Guillain-Barré syndrome (GBS) have been described [2-5]. The SARS-CoV-2 virus binds to the ACE2 receptor, which is expressed in glial cells, neurons, and endothelial-arterial smooth muscle cells of the brain [6]. Potential mechanisms of SARS-CoV-2 neurotropism include direct cell invasion, exaggerated inflammatory immune response, and innate or adaptive immune responses that may lead to endothelial injury [7]. Here, we present an unusual case report of late-onset GBS and right facial nerve palsy in a patient who had COVID-19 illness 2 months before neurological presentation.

\section{Case Report}

A 70-year-old woman presented to an outside hospital with rapidly progressive weakness and difficulty walking that had developed within $72 \mathrm{~h}$. The patient had a medical history of left carotid endarterectomy, obesity, rheumatoid arthritis (for which she was prescribed monthly infliximab [Remicade] injections), papillary thyroid cancer status after thyroidectomy, and atrial fibrillation (for which she was prescribed apixaban). Notably, 2 months earlier, the patient had been diagnosed with COVID-19, where PCR from a nasopharyngeal swab detected SARS-CoV-2, and a chest X-ray had confirmed pneumonia. During her COVID-19 illness, the patient had a productive cough, rhinorrhea, shortness of breath, diffuse body weakness, and fatigue. She did not require hospitalization and was not treated with any steroids or antivirals. Oxygen supplementation was used during recovery, but she reported complete respiratory resolution by the end of 4 weeks of self-quarantine. The patient made a full recovery from COVID-19 except for mild fatigue.

The patient had no preceding gastrointestinal illness, urinary tract, or other upper respiratory tract infections in the 2 months between COVID-19 diagnosis and her presentation with extremity weakness. The patient did not receive any immunizations during or after her COVID-19 illness. At the time of presentation, she had been off of infliximab therapy for 3 months, and her last injection of infliximab was 1 month before her COVID-19 illness.

Two months after her COVID-19 illness, the patient presented to the outside hospital with sudden onset of ascending numbness in her lower extremities followed by weakness, which progressed over 4 days. The patient lost her ability to walk, and she tested positive for SARS-CoV-2 IgG. GBS was suspected, and the patient had a spinal tap performed 7 days after her clinical presentation.

Cerebrospinal fluid (CSF) showed albuminocytological dissociation with white blood cell count $0 / \mathrm{mm}^{3}$, red blood cell count $2 / \mathrm{mm}^{3}$, and CSF protein $97 \mathrm{mg} / \mathrm{dL}$. Other abnormal laboratory results on admission included thyroid-stimulating hormone $22.91 \mathrm{uIU} / \mathrm{mL}$ (0.45-5.33 $\mathrm{uIU} / \mathrm{mL})$, thyroid peroxidase antibody $20 \mathrm{IU} / \mathrm{mL}(<9 \mathrm{IU} / \mathrm{mL})$, white blood count $13.13 \mathrm{~K} / \mathrm{uL}$ (3.8-10.6 K/uL), C-reactive protein $2.12 \mathrm{mg} / \mathrm{dL}(<0.5 \mathrm{mg} / \mathrm{dL})$, creatine phosphokinase 946 IU/L ( $<178 \mathrm{IU} / \mathrm{L})$, and erythrocyte sedimentation rate $43 \mathrm{~mm} / \mathrm{h}(0-20 \mathrm{~mm} / \mathrm{h})$. Ganglioside antibodies were not checked. The patient reported being compliant with her thyroid medications, and the medication dose needed to be adjusted. MRI of the brain and cervical spine was completed 5 days after symptom onset and showed no acute intracranial abnormality or nerve enhancement. The patient received intravenous immunoglobulin-G (IVIG) for the treatment of GBS. After 2 doses of IVIG treatment, she developed altered mental status and new-onset right lower motor neuron facial weakness. Due to concerns of a possible stroke, she was transferred to our hospital for further evaluation.

On arrival at our facility, the patient was encephalopathic but was able to follow simple commands. Cranial nerve examination showed decreased activation of the right upper and lower face indicative of lower motor neuron pattern of facial palsy without other cranial

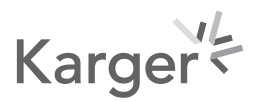



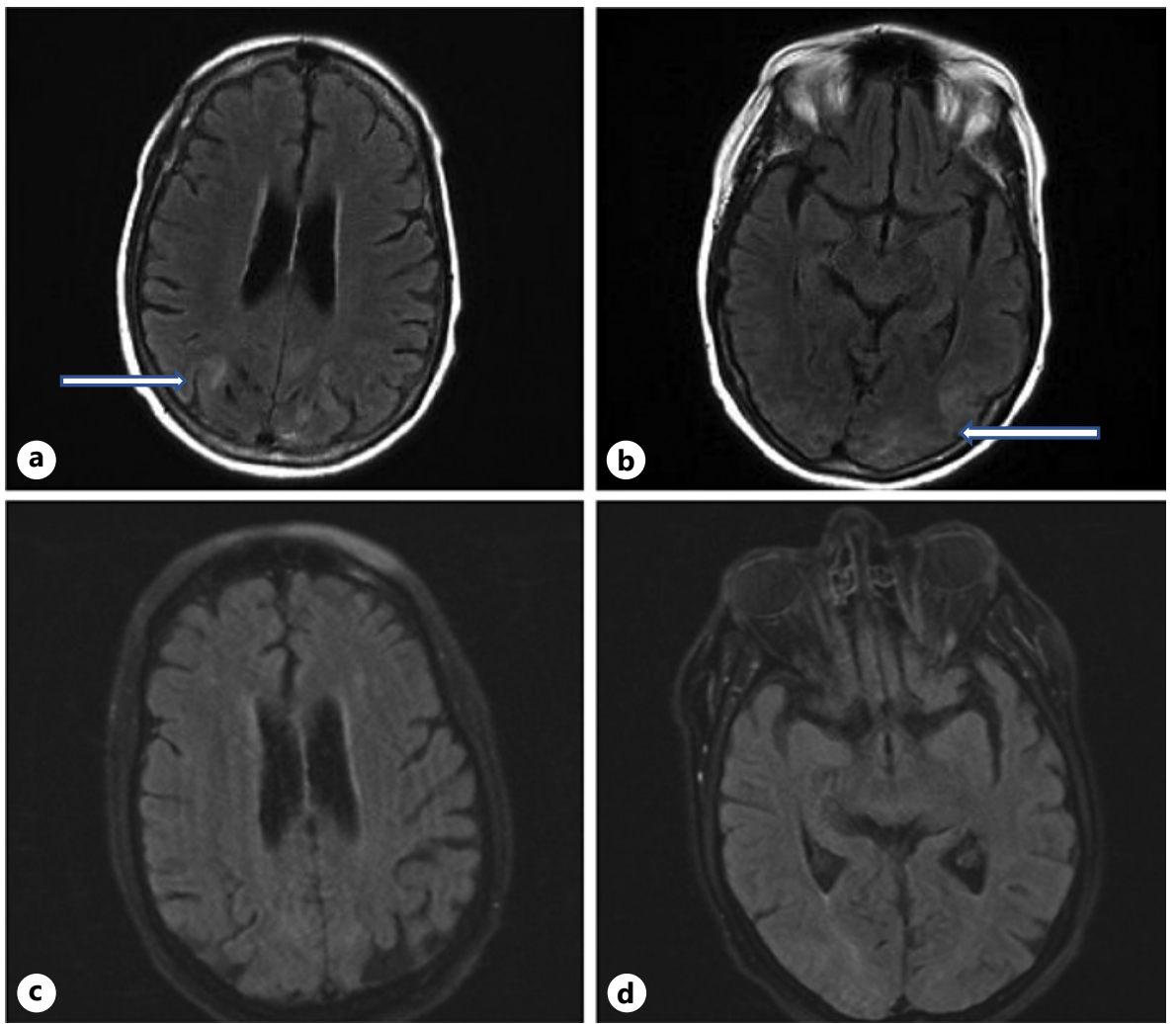

Fig. 1. Brain MRI axial FLAIR sequences before initiation of PLEX revealed T2 hyperintensities in the bilateral occipital and temporal lobes (white arrow) (a, b). Brain MRI axial FLAIR sequence after treatment of PLEX showed interval resolution of the T2 hyperintensities $(\mathbf{c}, \mathbf{d})$. FLAIR, fluid attenuation inversion recovery.

nerve abnormalities. She had 3-/5 strength in all proximal and distal upper and lower extremities. She was areflexic in the upper and lower extremities. The patient withdrew to noxious stimuli in all 4 extremities, but further sensory testing was limited by the patient's encephalopathy.

MRI of the brain repeated 7 days after symptom onset showed T2 hyperintensity of the bilateral parietal occipital lobes and left posterior temporal lobe correlating with posterior reversible encephalopathy syndrome (PRES) without DWI/ADC changes (Fig. 1a, b), which were presumed to be adverse effects from the IVIG treatment. Imaging did not demonstrate any $\mathrm{T} 2$ hyperintensity in the pontine region.

Electromyography performed 11 days after symptoms onset confirmed a severe subacute diffuse sensorimotor polyradiculoneuropathy with mixed features of acquired demyelination features (prolonged distal latencies, slowed conduction velocity, and conduction block) and axonal loss (online suppl. Table; for all online suppl. material, see www.karger.com/ doi/10.1159/000521245). Due to adverse effects from IVIG, she was started on plasmapheresis (PLEX). She received 5 rounds of PLEX every other day. Repeat MRI of the brain following 1 round of PLEX showed improvement of PRES (Fig. 1c, d).

Before discharge, the patient's mental status had improved. She demonstrated minimal improvement of her right facial weakness, motor strength, and sensation. The patient's motor strength after PLEX treatment had mildly improved to 4-/5 in all proximal and distal upper and lower extremity muscles. She was able to sit up with assistance, and her sensation to light touch was normal but reduced to pinprick in all 4 extremities. Vibratory sensation was absent, 
and proprioception was impaired distally in her toes. Physical and occupational therapists reported improvement in fine motor movements, such as the ability to feed herself. The patient was able to maintain a sitting position and was able to perform $25-49 \%$ of physical activity independently.

Physical therapy assessed the patient, and reported physical limitations were mostly due to truncal and lower extremity weakness. She was able to maintain upright sitting position and truncal stability for $10 \mathrm{~min}$ independently. She did however require maximal assistance of 2 people to initiate upright position. Standing and ambulation were deferred due to the significant truncal weakness.

The patient was discharged to subacute rehabilitation and continued to make progress with physical and occupational therapy. She was discharged home after 4 months. At the time of discharge from rehabilitation, the patient was walking with a walker. Per the last conversation with the patient by telephone, she is now walking independently without any assistance. She had complete resolution of her facial weakness shortly after she was discharged to rehabilitation, and she achieved complete recovery within about 6 months.

\section{Discussion}

Multiple instances of neurological involvement with SARS-CoV-2 infection have been reported since March 2020 [7], including several case reports, series, and review articles on GBS.3-5. Based on previous reports, GBS associated with COVID-19 is similar to GBS caused by more common infectious culprits such as Campylobacter jejuni (2-20 days), cytomegalovirus (4 weeks), and influenza A virus (3-30 days), with neurological symptoms appearing within 1-3 weeks after infection [8-10]. In a systematic review of 73 cases of GBS associated with COVID-19, neurological manifestations occurred within a median of 14 days [11]. In another review of GBS, the author noted that in most cases, patients manifested GBS-related symptoms in about 1-4 weeks after the diagnosis of COVID-19, except 2 patients who presented with GBS at the time of COVID-19 illness [12].

Our case is unique because the patient presented with late-onset GBS a full 2 months after her COVID-19 illness. She has made a complete recovery from her illness, and she had no neurological problems before having had COVID-19, which points to a possible post-infectious or immune-mediated GBS that occurred in response to the SARS-CoV-2 virus or its antibodies. A systematic review by Uncini et al. [13] revealed that the maximum time between onset of COVID-19 and the appearance of GBS symptoms for 36 patients was 28 days, which is markedly shorter than our case.

To our knowledge, the late onset of GBS (8 weeks) associated with SARS-CoV-2 has not been described. The pathogenesis involved in GBS is mainly a humoral-mediated rather than T-cell-mediated disorder [14]. Molecular mimicry and immune complex deposition have been described as possible immunopathogenic processes involved in the acute motor axonal neuropathy associated with certain microbes $[1,15,16]$. The most common clinical presentations of GBS associated with COVID-19 include paresthesia in the distal extremities, symmetric proximal and distal lower extremity weakness, acute quadriparesis, bilateral or unilateral facial nerve palsy, gait instability, and radicular pain [11,17].

The patient also noted to have high CPK levels which was thought to be secondary to metabolic cause such as hypothyroidism. However, there is evidence in the current medical literature that CPK alterations may be seen in up to $27 \%$ of patients with GBS in the first 4 weeks after symptoms' onset [18]. Moreover, patients presenting with CPKalterations usually have a form of AMSAN with reversible conduction failure (RCF). Although a concomitant subclinical endocrine myopathy remains in the differential diagnosis, it is also important to

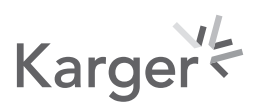


consider AMSAN as a possible diagnosis in this case given presence of axon loss more than the demyelination. Nonetheless, a single EMG study was performed, and RCF could not be excluded [19].

The patient presented clinical and neuroradiological features of PRES, although we believed that IVIG was the most likely etiology considering the temporal association. Nevertheless, PRES could have been the result of the same immune-mediated process driving GBS. Moreover, the patient has a history of autoimmune disease, which is a recognized risk factor for the development of PRES [20]. It is equally important to address the association of infliximab with GBS; however, it is less likely the cause in this case given the fact that the patient had her last infliximab dose 3 months prior to her neurological manifestations [21].

GBS in the context of COVID-19 could be related to a genetic predisposition for autoimmune disorders. A case report by Gigli et al. [22] explored the possible correlation between the IL-8 pathway as well as genetic predisposition in COVID-induced GBS. They reported a patient, like ours, who was infected with COVID several weeks prior to neurological deterioration. Cytokines and COVID antibodies were tested in the CSF as well as serum, and HLA subtypes were assessed. Their patient demonstrated several HLA alleles which have been shown to be associated with GBS including HLA-A33, DRB1*3:01. DQB1*5:01 [22].

Our patient also manifested features of unilateral facial paralysis. Given the absence of brainstem involvement from PRES or other vascular insults, the unilateral facial paralysis in our patient may have been related to an immune-mediated facial neuropathy as a separate sequela of post-COVID-19 illness with GBS. Several case reports and series have described a relationship between COVID-19 and facial paralysis with and without association with GBS [3, 17-19].

Given that SARS-CoV-2 PCR analysis of CSF was negative in a COVID-19 patient with cranial nerve involvement, facial neuropathy may be secondary to injury from pro-inflammatory cytokines rather than from the virus itself, with or without association with GBS [23, 24]. Nevertheless, though rare, unilateral facial palsy is described as a clinical manifestation of GBS, and blink reflex testing may reveal subclinical neurophysiological involvement in the contralateral nerve [25].

Given that our patient had taken a TNF inhibitor (infliximab) 3 months before her neurologic symptoms, it is important to address the possible correlation between TNF inhibition and GBS. There are very few published cases that have reported on this clinical situation; however, discontinuation of TNF inhibitor therapy has correlated with improvement in neurologic symptoms in most cases [26-28]. Our patient's GBS was unlikely to have been caused directly from her ongoing treatment, but it is possible that her GBS was related to immune reconstitution while her TNF inhibitors had recently been stopped.

This case highlights the need for vigilance regarding the possible development of GBS in COVID-19 patients even months after viral diagnosis and disease resolution. Physicians should be careful not to confuse a late manifestation of GBS with chronic fatigue after COVID-19 respiratory illness or chronic inflammatory demyelinating polyradiculoneuropathy.

\section{Acknowledgment}

The authors thank Karla D Passalacqua, Ph.D., at Henry Ford Hospital, for assistance with editing.

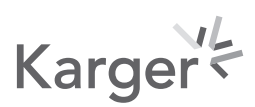


Taguchi et al.: Late-Onset AIDP after COVID-19 Infection

\section{Statement of Ethics}

Henry Ford Hospital does not require IRB approval for the case report. Written informed consent was obtained from the patient for publication of this case report and any accompanying images.

\section{Conflict of Interest Statement}

The authors declare that they have no conflicts of interest to declare.

\section{Funding Sources}

No grant or support was received.

\section{Author Contributions}

Meari Taguchi: conceptualization of the content; writing the preliminary version of the manuscript; reviewing and approving the final manuscript before submission. Kyle Bonner: conceptualization of the overall academic content; editing the final version and approving it before submission. Anza B. Memon: conceptualization of the academic content related to the topic; co-writing of the preliminary version; editing the final version and approving it before submission.

\section{Data Availability Statement}

All data generated or analyzed during this study are included in this article.

\section{References}

1 Sharifian-Dorche M, Huot P, Osherov M, Wen D, Saveriano A, Giacomini PS, et al. Neurological complications of coronavirus infection; a comparative review and lessons learned during the COVID-19 pandemic. J Neurol Sci. 2020 Oct 15;417:117085.

2 Zhao H, Shen D, Zhou H, Liu J, Chen S. Guillain-Barré syndrome associated with SARS-CoV-2 infection: causality or coincidence? Lancet Neurol. 2020 May;19(5):383-4.

3 Toscano G, Palmerini F, Ravaglia S, Ruiz L, Invernizzi P, Cuzzoni MG, et al. Guillain-Barré Syndrome Associated with SARS-CoV-2. N Engl J Med. 2020;382(26):2574-6.

4 Gutiérrez-Ortiz C, Méndez A, Rodrigo-Rey S, San Pedro-Murillo E, Bermejo-Guerrero L, Gordo-Mañas R, et al. Miller Fisher syndrome and polyneuritis cranialis in COVID-19. Neurology. 2020 Aug 4;95(5):e601-e605.

5 Sedaghat Z, Karimi N. Guillain Barre syndrome associated with COVID-19 infection: a case report. J Clin Neurosci. 2020 Jun;76:233-5.

6 Li YC, Bai WZ, Hashikawa T. The neuroinvasive potential of SARS-CoV2 may play a role in the respiratory failure of COVID-19 patients. J Med Virol. 2020;92(6):552-5.

7 Zhou Z, Kang H, Li S, Zhao X. Understanding the neurotropic characteristics of SARSCoV2: from neurological manifestations of COVID19 to potential neurotropic mechanisms. J Neurol. 2020 Aug;267(8):2179-84.

8 Rees JH, Soudain SE, Gregson NA, Hughes RA. Campylobacter jejuni infection and Guillain-Barré syndrome. N Engl J Med. 1995 Nov 23;333(21):1374-9.

9 Jacobs BC, Rothbarth PH, van der Mechq FG, Herbrink P, Schmitz PI, de Klerk MA, et al. The spectrum of antecedent infections in Guillain-Barré syndrome: a case-control study. Neurology. 1998;51(4):1110-5.

10 Lehmann HC, Hartung HP, Kieseier BC, Hughes RA. Guillain-Barré syndrome after exposure to influenza virus. Lancet Infect Dis. 2010 Sep;10(9):643-51. 
11 Abu-Rumeileh S, Abdelhak A, Foschi M, Tumani H, Otto M. Guillain-Barré syndrome spectrum associated with COVID-19: an up-to-date systematic review of 73 cases. J Neurol. 2020 Aug 25;268(4):1133-70.

12 Rahimi K. Guillain-Barre syndrome during COVID-19 pandemic: an overview of the reports. Neurol SciPMCID. 2020 Nov;41(11):3149PMC7464053-56.

13 Uncini A, Vallat JM, Jacobs BC. Guillain-Barré syndrome in SARS-CoV-2 infection: an instant systematic review of the first six months of pandemic. J Neurol Neurosurg Psychiatry. 2020 Oct;91(10):1105-10.

14 Soliven B. Animal models of autoimmune neuropathy. ILAR J. 2014;54:282-90.

15 Sudo M, Miyaji K, Späth PJ, Morita-Matsumoto K, Yamaguchi Y, Yuki N. Polyclonal IgM and IgA block in vitro complement deposition mediated by anti-ganglioside antibodies in autoimmune neuropathies. Int Immunopharmacol. 2016;40:11-5.

16 Kuwabara S. Guillain-Barré syndrome. Epidemiology, pathophysiology and management. Drugs. 2004;64: 597-610.

17 Ottaviani D, Boso F, Tranquillini E, Gapeni I, Pedrotti G, Cozzio S, et al. Giometto early Guillain-Barré syndrome in coronavirus disease 2019 (COVID-19): a case report from an Italian COVID-hospital B. Neurol Sci. 2020;41: 1351-4.

18 Hosokawa T, Nakajima H, Sawai T, Nakamura Y, Sano E, Tsukahara A, et al. Clinical features of Guillain-Barré syndrome patients with elevated serum creatine kinase levels. BMC Neurol. 2020 May 27;20(1):214.

19 Chareyre J, Hully M, Simonnet H, Musset L, Barnerias C, Kossorotoff M, et al. Acute axonal neuropathy subtype of Guillain Barré syndrome in a French pediatric series: Adequate follow-up may require repetitive electrophysiological studies. Eur J Paediatr Neurol. 2017 Nov;21(6):891-7.

20 Stetefeld HR, Lehmann HC, Fink GR, Burghaus L. Posterior reversible encephalopathy syndrome and stroke after intravenous immunoglobulin treatment in Miller-Fisher syndrome/Bickerstaff brain stem encephalitis overlap syndrome. J Stroke Cerebrovasc Dis. 2014 0ct;23(9):e423-5.

21 Patwala K, Crump N, De Cruz P. Guillain-Barré syndrome in association with antitumour necrosis factor therapy: a case of mistaken identity. BMJ Case Rep. 2017 Jul 5;2017:bcr2017219481.

22 Gigli GL, Vogrig A, Nilo A, Fabris M, Biasotto A, Curcio F, et al. HLA and immunological features of SARS-CoV2-induced Guillain-Barré syndrome. Neurol Sci. 2020 Dec;41(12):3391-4.

23 Codeluppi L, Venturelli F, Rossi J, Fasano A, Toschi G, Pacillo F, et al. Facial palsy during the COVID-19 pandemic. Brain Behav. 2021 Jan;11(1):e01939.

24 Lima MA, Silva MTT, Soares CN, Coutinho R, Oliveira HS, Afonso L, et al. Peripheral facial nerve palsy associated with COVID-19. Neurovirol. 2020 Dec;26(6):941-4.

25 Verma R, Chaudhari TS, Giri P. Unilateral facial palsy in Guillain-Barre syndrome (GBS): a rare occurrence. BM] Case Rep. 2012 Oct 19;2012:bcr2012007077.

26 Goh Y, Beh DLL, Makmur A, Somani J, Chan ACY. Pearls \& Oy-sters: Facial nerve palsy in COVID-19 infection. Neurology. 2020 Aug 25;95(8):364-7.

27 Patwala K, Crump N, De Cruz P. Guillain-Barré syndrome in association with antitumour necrosis factor therapy: a case of mistaken identity. BMJ Case Rep. 2017 Jul 5;2017:bcr2017219481.

28 Baer AN, Kwon HJ, Papadopoulos EJ, Siegel JN. Guillain-Barre' and Miller Fisher Syndromes Occurring with Tumor Necrosis Factor Antagonist Therapy. Arthritis Rheumatism. May 2006;54(5):1429-34. 